\title{
四种天然双异戊烯基黄酮的合成及其抑菌活性研究
}

\author{
杨金会* 谢一民冯尚彪左武标陈兵兵 \\ 马晓琴冯 尧
}

(宁夏大学天然气转化国家重点实验室培育基地 银川 750021)

\begin{abstract}
摘要 Abyssinone-V, 4'-hydroxy-6,3',5'-triprenylisoflavonone, abyssinone-VI 和 abyssinone-IV 都是从刺桐中分离出来的天 然双异戊烯基黄酮. 利用简单、温和的方法首次完成了这 4 个天然产物的全合成. 初步活性测试表明, 4 个化合物都具 有一定的抑菌活性，其中, abyssinone-IV 和 abyssinone-V 对金黄葡萄球菌和大肠杆菌都有良好的抑菌效果. 所有新化合 物的结构都经过 HRMS, ${ }^{1} \mathrm{H}$ NMR 和 ${ }^{13} \mathrm{C}$ NMR 确认.

关键词 异戊烯基黄酮; (土)-abyssinone-VI; (土)-abyssinone-IV; (土)-abyssinone-V; 4'-hydroxy-6,3',5'-triprenylisoflavonone; 全合成; 抑菌活性
\end{abstract}

\section{Total Synthesis and Antibacterial Activities of Four Natural Dual Prenylated Flavonoids}

\author{
Yang, Jinhui* \\ Xie, Yimin \\ Feng, Shangbiao \\ Zuo, Wubiao \\ Chen, Bingbing \\ Ma, Xiaoqin \\ Feng, Yao \\ (State Key Laboratory Cultivation Base of Natural Gas Conversion, Ningxia University, Yinchuan 750021)
}

\begin{abstract}
Abyssinone-V, 4'-hydroxy-6,3',5'-triprenylisoflavonone, abyssinone-VI and abyssinone-IV were all isolated from the genus erythrina. This study completed the first total synthesis of four natural prenylated flavanoids by using simple and facile approaches. The result of their in vitro antibacterial tests indicated that all of them displayed moderate bacteriostatic activities. The compounds abyssinone-IV and abyssinone-V showed remarkable antibacterial activities. The structures of all new compounds have been confirmed by HRMS, ${ }^{1} \mathrm{H}$ NMR and ${ }^{13} \mathrm{C}$ NMR spectra.
\end{abstract}

Keywords prenylated flavanoid; ( \pm )-abyssinone-VI; ( \pm )-abyssinone-IV; ( \pm )-abyssinone-V; 4'-hydroxy-6,3',5'-triprenylisoflavonone; total synthesis; antibacterial activity

天然黄酮化合物广泛存在于植物界, 数量众多, 结 构复杂, 并且具有许多重要的生理和药理活性, 如抗 菌 $^{[1]} 、$ 抗诱变 ${ }^{[2]}$ 、抗真菌 ${ }^{[3]}$ 、抗肿瘤活性 ${ }^{[4-6]}$ 等作用. 化 合物 $\mathbf{1} \sim \mathbf{4}$ (图 1) 是从刺桐(Erythrina) 类植物中分离出来 的 ${ }^{[7 \sim 10]}$. 刺桐家族广泛分布在热带、亚热带地区，我国 的华南及四川地区也有广泛栽培. 刺桐类植物中富含黄 酮类化合物, 本身也曾是一种民间药用植物. 但是, 从 自然界中分离出来的黄酮远远满足不了人们的需要, 因 此, 人工合成就显得尤为重要. 我们研究小组 ${ }^{[11 ~ 14]}$ 一直 开展黄酮化合物的研究, 已完成了一些天然异戊烯基取 代的黄酮化合物的合成. 在此基础上, 我们课题组完成
了这 4 个天然产物的合成, 并初步测试了目标化合物的 抑菌活性. 合成路线见 Scheme 1.

\section{1 结果与讨论}

\section{1 合成}

天然异戊烯基黄酮化合物 1 4 的合成路线如 Scheme 1 所示. 关键中间体化合物 $\mathbf{6}$ 的合成按照我们 ${ }^{[11]}$ 已报道方法, 化合物 $\mathbf{6}$ 在无水 $\mathrm{K}_{2} \mathrm{CO}_{3}$, 丙酮的条件下用 $\mathrm{MOMCl}\left(\mathrm{CH}_{3} \mathrm{OCH}_{2} \mathrm{Cl}\right)$ 保护羟基, 以 $90 \%$ 的产率得到化 合物 7. 2,4,6-三羟基苯乙酮 (8) 和 2,4-二羟基苯乙䣯(13)

\footnotetext{
*E-mail: yang_jh@nxu.edu.cn

Received May 27, 2013; revised June 13, 2013; published online June 25, 2013.

Project supported by the National Natural Science Foundation of China (No. 21162021) and the Program for New Century Excellent Talents in University (No. NCET-09-0860).

国家自然科学基金(No.21162021)、教育部 “新世纪优秀人才支持计划” (No. NCET-09-0860)资助项目.
} 
<smiles>CC(C)=CCc1cc(C2CC(=O)c3c(O)cc(O)cc3O2)cc(CC=C(C)C)c1O</smiles>

(士)-abyssinone-V (1)<smiles>CC(C)=CCc1cc(/C=C/C(=O)c2ccc(O)cc2O)cc(CC=C(C)C)c1O</smiles>

$( \pm)$-abyssinone-VI (3)<smiles>CC(C)=CCc1cc(C2CC(=O)c3c(cc(O)c(CC=C(C)C)c3O)O2)cc(CC=C(C)C)c1O</smiles>

4'-hydroxy-6, 3', 5' -triprenylisoflavonone (2)<smiles>CC(C)=CCc1cc(C2CC(=O)c3ccc(O)cc3O2)cc(CC=C(C)C)c1O</smiles>

$( \pm)$-abyssinone-IV (4)

图 1 化合物 1 4 的结构

Figure 1 The structures of compounds $1 \sim \mathbf{4}$

经选择性差基保护，分别以 $87 \%$ 的收率得到缩合前体 $\mathbf{9}$, 以 $90 \%$ 的收率得到缩合前体 14. 2,4,6-三差基苯乙酩(8) 的四氢呋喃溶液在无水 $\mathrm{K}_{2} \mathrm{CO}_{3}$ 存在下和异戊烯基溴作 用, 以 $34.9 \%$ 的产率得到单 C-异戊烯基化产物 $\mathbf{1 0}^{[14]}$. 化 合物 10 经选择性差基保护以 89\%的收率得到缩合前体 11. 这样, 我们完成了 4 个缩合前体的合成.

化合物 7 和化合物 9, 11, 14 在 $\mathrm{KOH}, \mathrm{H}_{2} \mathrm{O}-\mathrm{EtOH}$ $(V: V=2: 3)$ 的作用下缩合，分别得到查尔酮 $15(78 \%)$, $16(76 \%)$ 以及 $19(75 \%)$. 查尔酮 15 在乙醇中, 在 $\mathrm{NaOAc}$ 作用下的条件下, 以 $50 \%$ 的收率关环得到化合物 17 , 黄 烷酮 17 随后在甲醇 $(\mathrm{MeOH})$ 中, 在盐酸作用下, 脱去 MOM 保护基得到目标化合物 $( \pm)-\mathbf{1}$.

查尔酮 16 先脱去保护基, 再环化, 得到目标化合物 (土)-2. 查尔酮 19 直接脱去保护基, 得到目标化合物 (土)-3, 再环化, 可以得到目标化合物 $( \pm)-4$. 目标化合 物(土)-4 也可以先由查尔酮 19 先环化, 再脱去保护基得 到. 合成的目标产物 $\mathbf{1} \sim \mathbf{4}$ 波谱数据与文献一致 ${ }^{[7 \sim 10]}$.

\section{2 抑菌活性}

采用微量肉汤稀释法对化合物 1 4 进行了革兰氏 阴性细菌模式菌(大肠杆菌)和革兰氏阳性细菌模式菌 (金黄葡萄球菌)的抑菌活性测试, 结果如表 1 所示. 通 过药物的不同浓度梯度 $(128,64,32,16,8,4,2,1,0.5,0$ $\mu \mathrm{g} / \mathrm{mL}$ )对化合物的抑菌效果进行检测. 我们发现, 大肠 杆菌和金黄葡萄球菌在所设定的不同浓度的 2 号和 3 号 化合物与在空白溶剂中生长状况相差不大. 而化合物 $\mathbf{1}$ 和 4 却表现出很好的抑菌活性. 其中, 化合物 4 对大肠 杆菌和金黄葡萄球菌的抑菌率达到 $70 \%$ 时的最低浓度
分别为 32 和 $16 \mu \mathrm{g} / \mathrm{mL}$. 而化合物 1 则对金黄葡萄球菌 表现出很好的抑制活性，抑制率达 $83 \%$ 时的最低浓度为 $8 \mu \mathrm{g} / \mathrm{mL}$. 但在此浓度下它对大肠杆菌的抑制不明显. 提示该化合物可能以干扰细菌细胞壁中肽聚糖合成而 达到抑菌效果的, 其具体药理作用有待进一步研究.

表 1 化合物 1 4 的抑菌活性(抑制率 $/ \%)^{a}$

Table 1 Antibacterial activities (inhibition rate/\%) of compounds $1 \sim 4$

\begin{tabular}{ccc}
\hline Compd. & S. aureus & E. coli \\
\hline $\mathbf{1}$ & 83 & 80 \\
$\mathbf{2}$ & - & - \\
$\mathbf{3}$ & - & - \\
$\mathbf{4}$ & 70 & 70 \\
\hline${ }^{a}$ 抑菌率 $(\%)=($ 阳性对照 $\mathrm{OD}$ 值 - 试验 $\mathrm{OD}$ 值 $) /($ 阳性对照 $\mathrm{OD}$ 值一阴性对照
\end{tabular}
$\mathrm{OD}$ 值 $) \times 100$.

\section{2 结论}

本文以对着基苯甲醛、2,4-二羟基苯乙酮、2,4,6-三 羟基苯乙酮为原料，经过碳代异成烯基化、羟基保护、 羟醛缩合、环合和脱去保护基团等几步合成了 abyssinone-V, 4'-hydroxy-6,3',5'-triprenylisoflavonone, abyssinone-VI 和 abyssinone-IV 4 个天然黄酮化合物 $\mathbf{1} \sim \mathbf{4}$. 采用微量肉汤稀释法对化合物 $1 \sim 4$ 进行了革兰氏阴性 细菌模式菌(大肠杆菌)和革兰氏阳性细菌模式菌(金黄 葡萄球菌)的抑菌活性测试. 初步研究表明, 化合物 1 4 对革兰氏阴性细菌模式菌(大肠杆菌)和对革兰氏阳性 细菌模式菌(金黄葡萄球菌)具有良好的抑制活性. 其中, 化合物 2 和 3 抑菌效果明显, 最低抑制浓度 $8 \mu \mathrm{g} / \mathrm{mL}$, 有 一定的药理应用前景. 
<smiles></smiles><smiles>CC(=O)c1c(O)cc(O)cc1O</smiles><smiles>CCCc1c(O)cc(O)c(C(C)=O)c1O</smiles><smiles>COc1cc(OC)c(C(C)=O)c(O)c1OC</smiles><smiles>CC(=O)c1ccc(O)cc1O</smiles>

13<smiles>CCCCCCCC(=O)c1ccc(OC)cc1O</smiles>

14<smiles>CCCc1cc(C2CC(=O)c3ccc(O)cc3O2)cc(CCC)c1Cl</smiles>

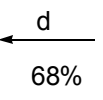<smiles>CCCc1cc(/C=C/C(=O)c2ccc(O)cc2O)cc(CCC)c1O</smiles>

e $\uparrow 78 \%$<smiles>CCCc1cc(C=O)cc(CCC)c1O</smiles>

16<smiles>CCCCC(C)(C)C(C)(C)C</smiles><smiles>CCCc1cc(C2CC(=O)c3c(cc(O)c(O)c3O)O2)cc(CCC)c1O</smiles><smiles>CC(C)=CC[C@H](C)C=[Po]</smiles><smiles>[13CH3]</smiles><smiles>CCCCCCCC</smiles><smiles>CCCc1cc(C2CC(=O)c3c(OC)cc(OC)cc3O2)cc(CCC)c1OC</smiles>

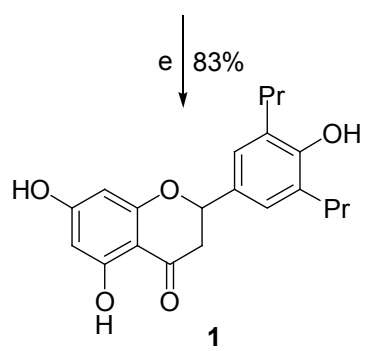

Reagents and conditions: (a) isoprenyl bromide, $\mathrm{KOH}, \mathrm{H}_{2} \mathrm{O}$; (b) anhydrous $\mathrm{K}_{2} \mathrm{CO}_{3}$, acetone, $\mathrm{MOMCl}$, reflux; (c) $\mathrm{KOH}-\mathrm{H}_{2} \mathrm{O}-\mathrm{EtOH}, \mathrm{N}_{2}$; (d) $\mathrm{EtOH}$, $\mathrm{NaOAc}$, reflux; (e) $\mathrm{MeOH}, \mathrm{HCl}$, reflux

\section{Scheme 1}

\section{3 实验部分}

\section{1 仪器与试剂}

NMR 用 Bruker AM-400 MHz 型核磁共振仪测定 $\left(\mathrm{CDCl}_{3}\right.$ 作溶剂, TMS 为内标); HRMS 用 Q-TOF 质谱仪 型号 Bruker maXis UHR-TOF; 昆山市超声仪器有限公 司 KQ-250DB 型数控超声波清洗器; 200 300 目及 $\mathrm{GF}_{254}$ 硅胶(青岛海洋化工厂生产); 试剂均为分析纯.

\section{2 实验方法}

4-差基-3,5-二异戊烯基苯甲醛(6)的合成参照文献 [11]，2,4,6-三羟基-3-异戊烯基苯乙酮(10)的合成参照文 献[14]，2-羟基-3-异戊烯基-4,6-二(甲氧甲氧基)苯乙酮 (11)的合成参照文献[14].

3.2.14-甲氧甲氧基-3,5-二异戊烯基苯甲醛(7)的合 成

将化合物 $6(258.0 \mathrm{mg}, 1.0 \mathrm{mmol})$ 溶于 $10 \mathrm{~mL}$ 干燥丙 
酮中, 搅拌下加入无水碳酸钾 $(304.0 \mathrm{mg}, 2.2 \mathrm{mmol})$, 加 热回流 $10 \mathrm{~min}$ 后, 缓慢滴加 $\mathrm{MOMCl}(176.0 \mathrm{mg}, 2.2$ $\mathrm{mmol}$ ), 继续搅拌 $30 \mathrm{~min}$ 后, 减压蒸除溶剂, 剩余物加 水溶解, 乙酸乙酯萃取 $(20 \mathrm{~mL} \times 3)$, 合并有机层并依次 用水洗, 饱和食盐水洗, 无水硫酸镁干燥, 过滤除去干 燥剂, 减压蒸除溶剂, 用 $V$ (乙酸乙酯 $): V($ 石油醚 $)=1$ : 10 为洗脱剂, 硅胶柱层析得无色油状物 7 (273.9 mg, 0.9 $\mathrm{mmol}$ ), 产率 90\%. ${ }^{1} \mathrm{H} \mathrm{NMR}\left(\mathrm{CDCl}_{3}, 400 \mathrm{MHz}\right) \delta: 9.89$ (s, $1 \mathrm{H}, \mathrm{CHO}), 7.56$ (s, 2H, H-2 and H-6), $5.27 \sim 5.31(\mathrm{~m}, 2 \mathrm{H}$, H-2' and H-2"), $5.01\left(\mathrm{~s}, 2 \mathrm{H}, \mathrm{OCH}_{2} \mathrm{O}\right) 3.61\left(\mathrm{~s}, 3 \mathrm{H}, \mathrm{OCH}_{3}\right)$, 3.43 (d, $J=7.2 \mathrm{~Hz}, 4 \mathrm{H}, \mathrm{H}-1^{\prime}$ and H-1"), 1.76 (s, 12H, H-4', H-4", H-5' and H-5");

\section{2 .2 2-差基-4, 6-二(甲氧甲氧基)苯乙酮(9)的合成}

将 2,4,6-三羟基苯乙酮(336.0 mg, $2.0 \mathrm{mmol}$ )溶于 20 $\mathrm{mL}$ 干燥的丙酮中, 剧烈搅拌下加入无水碳酸钾 $(1.4 \mathrm{~g}$, $10.1 \mathrm{mmol}$ ), 加热回流 $10 \mathrm{~min}$ 后, 缓慢滴加氯甲基甲基 醚(640.0 mg, $8.0 \mathrm{mmol})$, 继续摚拌回流 $2 \mathrm{~h}$, 冷却至室 温, 减压蒸去溶剂, 残余物直接用 $V$ (乙酸乙酯) : $V$ (石 油醚 $)=1: 10$ 为洗脱剂, 硅胶柱层析得淡黄色油状物 9 (460.8 mg, $1.8 \mathrm{mmol}$ ), 产率 87\%. ${ }^{1} \mathrm{H} \mathrm{NMR}\left(\mathrm{CDCl}_{3}, 400\right.$ $\mathrm{MHz}) \delta: 13.71$ (s, 1H, OH), 6.23 (d, $J=2.4 \mathrm{~Hz}, 1 \mathrm{H}, \mathrm{ArH})$, $6.21(\mathrm{~d}, J=2.4 \mathrm{~Hz}, 1 \mathrm{H}, \mathrm{ArH}), 5.23\left(\mathrm{~s}, 2 \mathrm{H}, \mathrm{OCH}_{2} \mathrm{O}\right), 5.14$ (s, 2H, $\left.\mathrm{OCH}_{2} \mathrm{O}\right), 3.49$ (s, $\left.3 \mathrm{H}, \mathrm{OCH}_{3}\right), 3.44$ (s, 3H, $\mathrm{OCH}_{3}$ ), $2.63\left(\mathrm{~s}, 3 \mathrm{H}, \mathrm{COCH}_{3}\right)$; HREIMS calcd for $\mathrm{C}_{12} \mathrm{H}_{16} \mathrm{O}_{6}$ 256.0947, found 256.0948

\section{2 .3 2-着基-4-甲氧甲氧基苯乙酮(14)的合成}

将 2,4-二羟基苯乙酮 $(308.0 \mathrm{mg}, 2.0 \mathrm{mmol}$ )溶于 20 $\mathrm{mL}$ 干燥的丙酮中, 剧烈搅拌下加入无水碳酸钾 $(690.0$ $\mathrm{mg}, 5.0 \mathrm{mmol}$ ), 加热回流 $10 \mathrm{~min}$ 后, 缓慢滴加氯甲基甲 基醚(320.0 mg, $4.0 \mathrm{mmol}$ ), 继续搅拌回流 $2 \mathrm{~h}$, 冷却至室 温, 减压蒸去溶剂, 残余物直接用 $V$ (乙酸乙酯) $: V$ (石 油醚 $)=1: 10$ 为洗脱剂, 硅胶柱层析得淡黄色油状物 14 (273.0 mg, $1.4 \mathrm{mmol})$, 产率 90\%. ${ }^{1} \mathrm{H} \mathrm{NMR}\left(\mathrm{CDCl}_{3}\right.$, $400 \mathrm{MHz}): 12.59$ (s, 1H, OH), 7.63 (d, $J=9.2 \mathrm{~Hz}, 1 \mathrm{H}$, $\operatorname{ArH}), 6.57$ (d, $J=2.4 \mathrm{~Hz}, 1 \mathrm{H}, \mathrm{ArH}), 6.53(\mathrm{dd}, J=8.8,2.4$ $\mathrm{Hz}, 1 \mathrm{H}, \mathrm{ArH}), 5.19$ (s, 2H, $\left.\mathrm{OCH}_{2} \mathrm{O}\right), 3.46\left(\mathrm{~s}, 3 \mathrm{H}, \mathrm{OCH}_{3}\right)$, $2.55\left(\mathrm{~s}, 3 \mathrm{H}, \mathrm{COCH}_{3}\right)$; HREIMS calcd for $\mathrm{C}_{10} \mathrm{H}_{12} \mathrm{O}_{4}$ 196.0736, found 196.0739

\subsubsection{2-羟基-4, $6,4^{\prime}$-三 (甲氧甲氧基)- $3^{\prime}, 5^{\prime}$-二异戊烯 基查尔酮(15)的合成}

将化合物 9 (307.2 mg, $1.2 \mathrm{mmol}$ )和化合物 7 (302.0 $\mathrm{mg}, 1.0 \mathrm{mmol}$ ) 溶解到 $2 \mathrm{~mL}$ 乙醇中, 冷却至 $0{ }^{\circ} \mathrm{C}$, 缓慢滴 加 $\mathrm{KOH}(2.8 \mathrm{~g}, 50.0 \mathrm{mmol})$ 的醇-水溶液 $7.5 \mathrm{~mL}(V: V=$ $3: 2)$, 氮气保护下 $0{ }^{\circ} \mathrm{C}$ 反应 $1 \mathrm{~h}$ 后, 自然恢复室温继续 反应 $24 \mathrm{~h}$, 将反应物倒入冰水中, 用 $3 \mathrm{~mol} / \mathrm{L}$ 的盐酸调
节 $\mathrm{pH}<2$, 用 $15 \mathrm{~mL}$ 乙酸乙酯萃取三次, 合并有机相并 依次水洗, 饱和食盐水洗, 无水硫酸镁干燥, 过滤除去 干燥剂, 减压蒸除溶剂, 剩余物用 $V$ (乙酸乙酯)： $V$ (石 油醚 $)=1: 6$ 为洗脱剂, 硅胶柱层析得黄色固体 15 (486.0 mg, $0.9 \mathrm{mmol})$, 产率 78\%. ${ }^{1} \mathrm{H} \mathrm{NMR}\left(\mathrm{CDCl}_{3}, 400\right.$ $\mathrm{MHz}) \delta: 13.92(\mathrm{~s}, 1 \mathrm{H}, \mathrm{OH}), 7.84(\mathrm{~d}, J=15.6 \mathrm{~Hz}, 1 \mathrm{H}$, H-6'), 7.75 (d, $J=15.6 \mathrm{~Hz}, 1 \mathrm{H}, \mathrm{H}-\beta), 7.44$ (d, $J=15.6 \mathrm{~Hz}$, $1 \mathrm{H}, \mathrm{H}-\alpha), 7.29$ (s, 2H, H-2 and 6), 6.31 (d, $J=2.0 \mathrm{~Hz}, 1 \mathrm{H}$, H-3'), 6.25 (d, J=2.4 Hz, 1H, H-5'), 5.32 5.29 (m, 2H, H-2' and $\mathrm{H}-2 "), 5.29\left(\mathrm{~s}, 2 \mathrm{H}, \mathrm{OCH}_{2} \mathrm{O}\right), 5.18\left(\mathrm{~s}, 2 \mathrm{H}, \mathrm{OCH}_{2} \mathrm{O}\right)$ $4.99\left(\mathrm{~s}, 2 \mathrm{H}, \mathrm{OCH}_{2} \mathrm{O}\right), 3.62\left(\mathrm{~s}, 3 \mathrm{H}, \mathrm{OCH}_{3}\right), 3.53(\mathrm{~s}, 3 \mathrm{H}$, $\mathrm{OCH}_{3}$ ), 3.47 (s, 3H, $\mathrm{OCH}_{3}$ ), 3.41 (d, J=7.2 Hz, 4H, H-1" and H-1"'); 1.79 (s, 12H, H-4", H-4"', H-5" and H-5"'); ${ }^{13} \mathrm{C}$ NMR $\left(\mathrm{CDCl}_{3}, 100 \mathrm{MHz}\right) \delta: 192.7,167.2,163.2,159.7$, $155.9,142.7,135.3,033.1,131.4,127.8,126.1,122.2$, 107.3, 99.8, 97.3, 94.8, 94.5, 93.9, 93.8, 57.4, 56.6, 56.3, $32.9,28.5,25.7,17.8$; HREIMS calcd for $\mathrm{C}_{31} \mathrm{H}_{40} \mathrm{O}_{8}$ 541.27959 , found 541.27972

$3.2 .55,7,4^{\prime}$-三(甲氧甲氧基)-3',5'-二异戊烯基黄烷酮 (17) 的合成

将化合物 15 (216.0 mg, $0.4 \mathrm{mmol}$ )溶解到 $7 \mathrm{~mL}$ 无水 乙醇中, 加入一滴水和无水醋酸钠 $(656.0 \mathrm{mg}, 8.0 \mathrm{mmol})$, 加热回流 $24 \mathrm{~h}$ 后, 冷却至室温, 加入少量水, 用 $20 \mathrm{~mL}$ 乙酸乙酯萃取 3 次, 合并有机层并依次用水洗, 饱和食 盐水洗, 无水硫酸镁干燥, 过滤除去干燥剂, 减压蒸除 溶剂, 剩余物用 $V($ 乙酸乙酯 $): V($ 石油醚 $)=1: 10$ 为洗 脱剂, 硅胶柱层析得淡黄色液体 17 (124.0 mg, 0.2 $\mathrm{mmol})$, 产率 50\%. ${ }^{1} \mathrm{H} \mathrm{NMR}\left(\mathrm{CDCl}_{3}, 400 \mathrm{MHz}\right) \delta: 7.12(\mathrm{~s}$, $2 \mathrm{H}, \mathrm{H}-2^{\prime}$ and 6'), 6.42 (d, $\left.J=2.4 \mathrm{~Hz}, 1 \mathrm{H}, \mathrm{ArH}\right), 6.39$ (d, $J=2.0 \mathrm{~Hz}, 1 \mathrm{H}, \mathrm{ArH}), 5.32 \sim 5.29$ (m, 2H, H-2" and 2"'), $5.25\left(\mathrm{~s}, 2 \mathrm{H}, \mathrm{OCH}_{2} \mathrm{O}\right), 5.14\left(\mathrm{~s}, 2 \mathrm{H}, \mathrm{OCH}_{2} \mathrm{O}\right), 4.95(\mathrm{~s}, 2 \mathrm{H}$, $\left.\mathrm{OCH}_{2} \mathrm{O}\right), 3.60\left(\mathrm{~s}, 3 \mathrm{H}, \mathrm{OCH}_{3}\right), 3.52\left(\mathrm{~s}, 3 \mathrm{H}, \mathrm{OCH}_{3}\right), 3.46(\mathrm{~s}$, $3 \mathrm{H}, \mathrm{OCH}_{3}$ ), 3.41 (d, $J=7.2 \mathrm{~Hz}, 4 \mathrm{H}, \mathrm{H}-1$ " and H-1"'), 3.05 (dd, $J=13.6,16.8 \mathrm{~Hz}, 1 \mathrm{H}, \mathrm{H}-3 \mathrm{ax}), 2.81$ (dd, $J=2.8,16.4$ Hz, 1H, H-3eq), 1.74 (s, 6H, H-4" and H-5"), 1.71 (s, 6H, $\mathrm{H}-4$ "' and $\left.\mathrm{H}-5{ }^{\prime \prime}\right) ;{ }^{13} \mathrm{C}$ NMR $\left(100 \mathrm{MHz}, \mathrm{CDCl}_{3}\right) \delta$ : 189.3, $164.5,163.0,159.3,154.0,135.2,134.3,132.8,125.5$, 122.4, 107.1, 99.7, 97.8, 97.2, 94.8, 93.9, 57.3, 56.4, 56.3, 45.5, 28.7, 25.6, 17.8; HREIMS calcd for $\mathrm{C}_{31} \mathrm{H}_{40} \mathrm{O}_{8}$ 541.27959 , found 541.27972 .

\section{2 .9 (土)-Abyssinone-V (1) 的合成}

将化合物 17 (61.2 mg, $0.15 \mathrm{mmol}$ )溶解到 $2 \mathrm{~mL}$ 甲醇 中, 搅拌下加入 $3 \mathrm{~mol} / \mathrm{L}$ 盐酸 $1 \mathrm{~mL}$, 加热回流 $30 \mathrm{~min}$, 向溶液中加入 $1 \mathrm{~mL}$ 水, 用 $20 \mathrm{~mL}$ 乙酸乙酯萃取 3 次, 合 并有机层并依次水洗, 饱和食盐水洗, 无水硫酸镁干燥, 
过滤除去干燥剂, 减压蒸除溶剂, 剩余物用 $V($ 乙酸乙 酯)： $V$ (石油醚 $)=1: 10$ 为洗脱剂, 硅胶柱层析得黄色 粘稠液体 1 (40.0 mg, $0.1 \mathrm{mmol})$ 产率 $83 \%$. ${ }^{1} \mathrm{H}$ NMR $\left(\mathrm{CDCl}_{3}, 400 \mathrm{MHz}\right) \delta: 12.07$ (s, 1H, OH), 7.05 (s, 2H, H-2' and H-6'), 5.99 (d, $J=2.0 \mathrm{~Hz}, 1 \mathrm{H}, \mathrm{ArH}), 5.98$ (d, $J=2.0$ $\mathrm{Hz}, 1 \mathrm{H}, \mathrm{ArH}), 5.33$ (dd, $J=2.8,13.2 \mathrm{~Hz}, 1 \mathrm{H}, \mathrm{H}-2), 5.27 \sim$ 5.30 (m, 2H, H-2" and H-2"'), 3.37 (d, J=7.2 Hz, 4H, H-1" and $\mathrm{H}-1 " '), 3.12$ (dd, $J=13.2,17.2 \mathrm{~Hz}, 1 \mathrm{H}, \mathrm{H}-3 \mathrm{ax}$ ), 2.77 (dd, $J=2.8,17.2 \mathrm{~Hz}, 1 \mathrm{H}, \mathrm{H}-3 \mathrm{eq}), 1.77$ (s, 12H, H-4" and H-4"', H-5" and H-5"'); ${ }^{13} \mathrm{C}$ NMR $\left(\mathrm{CDCl}_{3}, 100 \mathrm{MHz}\right) \delta$ : $196.5,164.8,164.2,163.3,134.8,129.5,127.5,126.0$, 121.4, 103.0, 96.5, 95.5, 43.0, 29.6, 25.8, 17.8; HREIMS calcd for $\mathrm{C}_{25} \mathrm{H}_{28} \mathrm{O}_{5} 409.20095$, found 409.20154

3.2.10 2-羟基-4,6,4'-三(甲氧甲氧基)-5, 3', 5'-三异戊 烯基查尔酮(16)的合成

将化合物 11 (296.7 mg, $0.91 \mathrm{mmol}$ )和化合物 7 $\left(274.8 \mathrm{mg}, 0.91 \mathrm{mmol}\right.$ )溶解到 $2 \mathrm{~mL}$ 乙醇中, 冷却至 $0{ }^{\circ} \mathrm{C}$, 缓慢滴加 $\mathrm{KOH}(2.54 \mathrm{~g}, 50 \mathrm{mmol})$ 的醇-水溶液 $6.7 \mathrm{~mL}$ $(V: V=3: 2)$, 氮气保护下 $0{ }^{\circ} \mathrm{C}$ 反应 $1 \mathrm{~h}$ 后, 自然恢复室 温继续反应 $24 \mathrm{~h}$, 将反应物倒入冰水中, 用 $3 \mathrm{~mol} / \mathrm{L}$ 的 盐酸调节 $\mathrm{pH}<2$, 用乙酸乙酯 $(25 \mathrm{~mL} \times 3)$ 萃取, 合并有 机相并依次水洗, 饱和食盐水洗, 无水硫酸镁干燥, 过 滤除去干燥剂, 减压蒸除溶剂, 剩余物用 $V($ 乙酸乙 酯) $: V$ (石油醚) $=1: 6$ 为洗脱剂, 硅胶柱层析得黄色液 体 16 (439.6 mg, $0.7 \mathrm{mmol})$, 产率 76\%. ${ }^{1} \mathrm{H} \mathrm{NMR}\left(\mathrm{CDCl}_{3}\right.$, $400 \mathrm{MHz}) \delta: 1.69$ (s, 3H, $\left.\mathrm{CH}_{3}\right), 1.73\left(\mathrm{~s}, 6 \mathrm{H}, \mathrm{CH}_{3}\right), 1.77$ (s, $\left.6 \mathrm{H}, \mathrm{CH}_{3}\right), 1.79\left(\mathrm{~s}, 3 \mathrm{H}, \mathrm{CH}_{3}\right), 3.39$ (d, $J=7.2 \mathrm{~Hz}, 2 \mathrm{H}$, H-1"'), 3.46 (d, $J=7.6 \mathrm{~Hz}, 4 \mathrm{H}, \mathrm{H}-1$ ' and H-1"), 3.50 (s, 3H, $\left.\mathrm{OCH}_{3}\right), 3.52\left(\mathrm{~s}, 3 \mathrm{H}, \mathrm{OCH}_{3}\right), 3.62\left(\mathrm{~s}, 3 \mathrm{H}, \mathrm{OCH}_{3}\right), 4.99$ (s, $\left.2 \mathrm{H}, \mathrm{OCH}_{2}\right), 5.20\left(\mathrm{~m}, 1 \mathrm{H}, \mathrm{H}-2^{\prime}\right), 5.28\left(\mathrm{~s}, 2 \mathrm{H}, \mathrm{OCH}_{2}\right), 5.30$ (s, 2H, $\mathrm{OCH}_{2}$ ), 5.32 (s, m, 2H, H-2"' and H-2"), 6.41 (s, 1H, H-6 or H-8), 7.28 (s, 2H, H-2 and H-6), 7.74 (d, $J=$ $15.6 \mathrm{~Hz}, 1 \mathrm{H}, \mathrm{H}-\alpha), 7.82$ (d, $J=16.0 \mathrm{~Hz}, 1 \mathrm{H}, \mathrm{H}-\beta), 13.85$ (s, $1 \mathrm{H}, \mathrm{OH}) ;{ }^{13} \mathrm{C}$ NMR $\left(\mathrm{CDCl}_{3}, 100 \mathrm{MHz}\right) \delta: 17.7,17.8$, 21.6, 25.7, 28.6, 56.2, 56.6, 57.5, 92.0, 93.8, 95.1, 99.8, $107.6,111.8,122.2,122.4,123.5,126.5,129.2,131.4$, $133.2,135.3,142.3,155.8,158.0,160.5,163.6,193.2$. HREIMS calcd for $\mathrm{C}_{36} \mathrm{H}_{49} \mathrm{O}_{8} 609.3451$, found 609.3427

3.2.112,4,6,4'-四(羟基)-5, 3',5'-三异戊烯基查尔酮 (18)的合成

将化合物 16 (150.7 mg, $0.24 \mathrm{mmol}$ )溶解到 $2 \mathrm{~mL}$ 甲 醇中, 搅拌下加入 $3 \mathrm{~mol} / \mathrm{L}$ 盐酸 $1 \mathrm{~mL}$, 加热回流 $10 \mathrm{~min}$, 向溶液中加入 $1 \mathrm{~mL}$ 水, 用 $20 \mathrm{~mL}$ 乙酸乙酯萃取 3 次, 合 并有机层并依次水洗, 饱和食盐水洗, 无水硫酸镁干燥, 过滤除去干燥剂, 减压蒸除溶剂, 剩余物用 $V($ 乙酸乙
酯)： $V$ (石油醚 $)=1: 10$ 为洗脱剂, 硅胶柱层析得黄色 固体 18 (124.0 mg, $0.25 \mathrm{mmol})$, 产率 80\%. ${ }^{1} \mathrm{H}$ NMR ( $\left.\mathrm{CDCl}_{3}, 400 \mathrm{MHz}\right) \delta: 1.77$ (s, 6H, $\left.\mathrm{CH}_{3}-4 " '\right), 1.82(\mathrm{~s}, 12 \mathrm{H}$, $\left.\mathrm{CH}_{3}-4 "\right), 3.35$ (d, $\left.J=7.2 \mathrm{~Hz}, 2 \mathrm{H}, \mathrm{H}-1{ }^{\prime \prime}\right), 3.39$ (d, $J=6.8$ $\mathrm{Hz}, 4 \mathrm{H}, \mathrm{H}-1{ }^{\prime \prime}$ and $\left.\mathrm{H}-1^{\prime}\right), 5.28$ (t, 1H, H-2"'), 5.32 (m, 3H, H-2', H-2"), 5.73 (s, 1H, H-8), 7.28 (s, 2H, H-2 and H-6), 7.77 (d, $J=15.6 \mathrm{~Hz}, 1 \mathrm{H}, \mathrm{H}-\alpha), 7.81$ (d, $J=15.6 \mathrm{~Hz}, 1 \mathrm{H}$, $\mathrm{H}-\beta) ;{ }^{13} \mathrm{C}$ NMR $\left(\mathrm{CDCl}_{3}, 100 \mathrm{MHz}\right) \delta: 18.1,21.7,25.9$, 29.6, 95.7, 105.6, 105.8, 121.5, 124.1, 127.6, 128.8, 135.1, 136.3, 144.1, 155.3, 161.0, 162.5, 192.9. HREIMS calcd for $\mathrm{C}_{30} \mathrm{H}_{35} \mathrm{O}_{5} 475.2484$, found 475.2475 .

3.2.12 5,7,4'-三(羟基)-6,3',5'-三异戊烯基黄烷酮(2) 的合成

将化合物 18 (99.2 mg, $0.2 \mathrm{mmol})$ 溶解到 $7 \mathrm{~mL}$ 无水 乙醇中, 加入一滴水和无水醋酸钠 $(164.0 \mathrm{mg}, 2.0 \mathrm{mmol})$, 加热回流 $24 \mathrm{~h}$ 后, 冷却至室温, 加入少量水, 用 $20 \mathrm{~mL}$ 乙酸乙酯萃取 3 次, 合并有机层并依次用水洗, 饱和食 盐水洗, 无水硫酸镁干燥, 过滤除去干燥剂, 减压蒸除 溶剂, 剩余物用 $V($ 乙酸乙酯 $): V($ 石油醚 $)=1: 10$ 为洗 脱剂, 硅胶柱层析得淡黄色粘稠液体 $2(78.9 \mathrm{mg}, 0.16$ $\mathrm{mmol})$, 产率 60\%. ${ }^{1} \mathrm{H} \mathrm{NMR}\left(\mathrm{CDCl}_{3}, 400 \mathrm{MHz}\right) \delta: 1.74$ (s, $\left.6 \mathrm{H}, \mathrm{CH}_{3}-44^{\prime \prime}\right), 1.80$ (s, 12H, $\left.\mathrm{CH}_{3}-4 "\right), 2.80$ (dd, $J=14.5,2.5$ $\mathrm{Hz}, 1 \mathrm{H}, \mathrm{H}-3$ ), 3.06 (dd, $J=14.5,2.5 \mathrm{~Hz}, 1 \mathrm{H}, \mathrm{H}-3), 3.30$ (d, $\left.J=6 \mathrm{~Hz}, 2 \mathrm{H}, \mathrm{H}-1{ }^{\prime \prime}\right), 3.38$ (d, $J=6.5 \mathrm{~Hz}, 4 \mathrm{H}, \mathrm{H}-1 "$ and H-1'), 5.20 (t, $\left.J=6.5 \mathrm{~Hz}, 1 \mathrm{H}, \mathrm{H}-2{ }^{\prime \prime}\right), 5.32$ (m, 3H, H-2', H-2"), 5.50 (s, 1H, OH-4), 6.02 (s, 1H, H-8), 6.05 (br s, 1H, OH-7), 7.05 (s, 2H, H-2' and H-6'), 12.02 (s, 1H, OH-5); ${ }^{13} \mathrm{C} \mathrm{NMR}\left(\mathrm{CDCl}_{3}, 100 \mathrm{MHz}\right) \delta: 17.9,21.8,25.8$, 29.7, 43.2, 79.1, 96.7, 103.2, 106.0, 121.5, 125.7, 127.4, 130.2, 134.8, 135.0, 153.1, 159.9, 162.3, 163.6, 196.4 . HREIMS calcd for $\mathrm{C}_{30} \mathrm{H}_{35} \mathrm{O}_{5} 475.2484$, found 475.2481.

3.2.132-羟基-4,4'-二(甲氧甲氧基)- $3^{\prime}, 5^{\prime}$-二异成烯基 查尔酮(19)的合成

将化合物 7 (362.4 mg, $1.2 \mathrm{mmol}$ )和化合物 14 (195.0 $\mathrm{mg}, 1.0 \mathrm{mmol}$ ) 溶解到 $2 \mathrm{~mL}$ 乙醇中, 冷却至 $0{ }^{\circ} \mathrm{C}$, 缓慢滴 加 $\mathrm{KOH}(2.8 \mathrm{~g}, 50.0 \mathrm{mmol})$ 的醇-水溶液 $6.7 \mathrm{~mL}(V: V=$ $3: 2)$, 氮气保护下 $0{ }^{\circ} \mathrm{C}$ 反应 $1 \mathrm{~h}$ 后, 自然恢复室温继续 反应 $24 \mathrm{~h}$, 将反应物倒入冰水中, 用 $3 \mathrm{~mol} / \mathrm{L}$ 的盐酸调 节 $\mathrm{pH}<2$, 用 $15 \mathrm{~mL}$ 乙酸乙酯萃取 3 次, 合并有机相并 依次水洗, 饱和食盐水洗, 无水硫酸镁干燥, 过滤除去 干燥剂, 减压蒸除溶剂, 剩余物用 $V$ (乙酸乙酯) $: V$ (石 油醚 $)=1: 8$ 为洗脱剂, 硅胶柱层析得黄色液体 19 (351.0 mg, $0.7 \mathrm{mmol})$, 产率 75\%. ${ }^{1} \mathrm{H} \mathrm{NMR}\left(\mathrm{CDCl}_{3}, 400\right.$ MHz) $\delta$ : 13.35 (s, 1H, OH), 7.85 (d, $\left.J=8.8 \mathrm{~Hz}, 1 \mathrm{H}, \mathrm{H}-6^{\prime}\right)$, $7.83(\mathrm{~d}, J=15.6 \mathrm{~Hz}, 1 \mathrm{H}, \mathrm{H}-\beta), 7.46$ (d, $J=15.6 \mathrm{~Hz}, 1 \mathrm{H}$, 
H- $\alpha$ ), 7.32 (s, 2H, H-2 and H-6), 6.64 (d, $J=2.8 \mathrm{~Hz}, 1 \mathrm{H}$, H-3'), 6.61 (dd, $\left.J=8.8,2.4 \mathrm{~Hz}, 1 \mathrm{H}, \mathrm{H}-5{ }^{\prime}\right), 5.32 \sim 5.28$ (m, $2 \mathrm{H}, \mathrm{H}-2^{\prime}$ and $\left.\mathrm{H}-2^{\prime \prime}\right), 5.28\left(\mathrm{~s}, 2 \mathrm{H}, \mathrm{OCH}_{2} \mathrm{O}\right), 4.99(\mathrm{~s}, 2 \mathrm{H}$, $\left.\mathrm{OCH}_{2} \mathrm{O}\right), 3.62\left(\mathrm{~s}, 3 \mathrm{H}, \mathrm{OCH}_{3}\right), 3.49\left(\mathrm{~s}, 3 \mathrm{H}, \mathrm{OCH}_{3}\right), 3.40$ (d, $J=6.8 \mathrm{~Hz}, 4 \mathrm{H}, \mathrm{H}-1 "$ and H-1"'), 1.78 (s, 6H, H-4" and

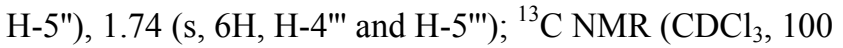
MHz) $\delta: 192.1,166.1,163.4,156.3,144.7,135.6,133.3$, $131.2,130.8,128.2,122.2,119.0,114.9,108.0,103.9$, 99.8, 93.9, 60.3, 57.5, 56.3, 28.7, 25.7, 17.9; HREIMS calcd for $\mathrm{C}_{29} \mathrm{H}_{36} \mathrm{O}_{6} 481.25846$, found 481.25870.

\subsubsection{4 (土)-Abyssinone-VI (3) 的合成}

将化合物 19 (99.4 mg, $0.2 \mathrm{mmol}$ )溶解到 $2 \mathrm{~mL}$ 甲醇 中, 搅拌下加入 $3 \mathrm{~mol} / \mathrm{L}$ 盐酸 $1 \mathrm{~mL}$, 加热回流 $10 \mathrm{~min}$, 向溶液中加入 $1 \mathrm{~mL}$ 水, 用 $20 \mathrm{~mL}$ 乙酸乙酯萃取 3 次, 合 并有机层并依次水洗, 饱和食盐水洗, 无水硫酸镁干燥, 过滤除去干燥剂, 减压蒸除溶剂, 剩余物用 $V($ 乙酸乙 酯) : $V$ (石油醚 $)=1 ： 10$ 为洗脱剂, 硅胶柱层析得黄色 液体 3 (66.4 mg, $0.2 \mathrm{mmol})$, 产率 78\%. ${ }^{1} \mathrm{H} \mathrm{NMR}\left(\mathrm{CDCl}_{3}\right.$, $400 \mathrm{MHz}) \delta$ : 13.59 (s, 1H, OH), 7.85 (d, J=7.6 Hz, 1H, H-6'), 7.79 (d, $J=15.2 \mathrm{~Hz}, 1 \mathrm{H}, \mathrm{H}-\beta), 7.43$ (d, $J=15.2 \mathrm{~Hz}$, $1 \mathrm{H}, \mathrm{H}-\alpha), 7.29$ (s, 2H, H-2 and H-6), 6.46 (dd, $J=2.4,11.2$ Hz, 1H, H-5'), 6.43 (d, $J=2.4$ Hz, 1H, H-3'), 5.28 5.32 (m, 2H, H-2" and H-2"'), 3.39 (d, $J=6.8 \mathrm{~Hz}, 4 \mathrm{H}, \mathrm{H}-1$ " and H-1"'), 1.82 (s, 12H, H-4", H-4"', H-5" and H-5"'); ${ }^{13} \mathrm{C}$ NMR $\left(\mathrm{CDCl}_{3}, 100 \mathrm{MHz}\right) \delta$ : 192.0, 166.2, 162.6, 155.6, $145.3,135.2,131.9,128.8,127.7,126.9,121.3,117.4$, 107.6, 103.6, 29.6, 27.0, 25.8, 17.9; HREIMS calcd for $\mathrm{C}_{25} \mathrm{H}_{28} \mathrm{O}_{5}$ 393.20604, found 393.20660.

\subsubsection{5 (士)-Abyssinone-IV (4)的合成}

将化合物 3 (55.6 mg, $0.1 \mathrm{mmol}$ ) 溶解到 $7 \mathrm{~mL}$ 无水乙 醇中, 加入一滴水和无水醋酸钠 $(82.0 \mathrm{mg}, 1.0 \mathrm{mmol})$, 加 热回流 $24 \mathrm{~h}$ 后, 冷却至室温, 加入少量水, 用 $20 \mathrm{~mL}$ 乙 酸乙酯萃取 3 次, 合并有机层并依次用水洗, 饱和食盐 水洗, 无水硫酸镁干燥, 过滤除去干燥剂, 减压蒸除溶 剂, 剩余物用 $V$ (乙酸乙酯 $): V($ 石油醚 $)=1: 10$ 为洗脱 剂, 硅胶柱层析得淡黄色粘稠液体 4 (48.9 mg, 0.1 $\mathrm{mmol})$, 产率 68\%. ${ }^{1} \mathrm{H} \mathrm{NMR}\left(\mathrm{CDCl}_{3}, 400 \mathrm{MHz}\right) \delta: 12.69$ (s, 1H, OH), 7.86 (d, $J=8.8 \mathrm{~Hz}, 1 \mathrm{H}, \mathrm{H}-5), 7.07$ (s, 2H, H-2' and H-6'), 6.55 (d, $J=2.4 \mathrm{~Hz}, 1 \mathrm{H}, \mathrm{ArH}), 6.53$ (d, $J=$ $2.4 \mathrm{~Hz}, 1 \mathrm{H}, \mathrm{ArH}), 5.35$ (dd, $J=2.8,13.6 \mathrm{~Hz}, 1 \mathrm{H}, \mathrm{H}-2)$, $5.32 \sim 5.29$ (m, 2H, H-2" and H-2"'), 3.37 (d, $J=7.2 \mathrm{~Hz}$, $4 \mathrm{H}, \mathrm{H}-1$ " and H-1"'), 3.12 (dd, $J=13.6,16.4 \mathrm{~Hz}, 1 \mathrm{H}$, H-3ax), 2.79 (dd, $J=2.8,16.4 \mathrm{~Hz}, 1 \mathrm{H}, \mathrm{H}-3 \mathrm{eq}), 1.81$ (s, 12H, H-4", H-4"', H-5" and H-5"'); ${ }^{13} \mathrm{C}$ NMR $\left(\mathrm{CDCl}_{3}, 100\right.$ MHz) $\delta: 191.8,165.0,163.8,163.0,153.3,134.8,133.3$,
$130.0,129.3,127.5,126.1,121.5,115.9,114.8,112.5$, $110.5,103.4,80.0,44.0,29.7,26.2,25.8,17.8$; HREIMS calcd for $\mathrm{C}_{25} \mathrm{H}_{28} \mathrm{O}_{4}$ 393.20604, found 393.20639.

\section{3 抑菌活性测定}

\subsection{1 实验材料}

抗菌药物咜存液制备：抗菌药物咜存液浓度 1280 $\mu \mathrm{g} / \mathrm{mL}$.

LB 液体培养基：胰化蛋白胨 $10 \mathrm{~g}$ ，酵母提取物 $5 \mathrm{~g}$, $\mathrm{NaCl} 10 \mathrm{~g}$ ，水溶解后，用 $5 \mathrm{~mol} / \mathrm{L}$ 的 $\mathrm{NaOH}$ 调 $\mathrm{pH}$ 至 7.5, 加蒸馏水定容到 $1 \mathrm{~L}$, 在 $103 \mathrm{kPa}\left(1.05 \mathrm{~kg} / \mathrm{cm}^{2}\right)$ 高压灭菌 $20 \mathrm{~min}$.

测试菌种：大肠杆菌标准株(抗生素测定用); 金黄 色葡萄球菌标准株(抗生素测定用).

\subsection{2 实验方法}

MIC 板制备: 无菌操作，将倍比稀释后不同浓度的 抗菌药物溶液分别加到灭菌的 100 孔细菌生长曲线测定 板中，第 1 至第 9 孔加药液，每孔 $10 \mu \mathrm{L}$ ，第 10 孔加药 品溶剂作为生长对照, 冰冻干燥后密封, $-20{ }^{\circ} \mathrm{C}$ 以下保 存备用. 每样设定 2 个平行样; 采取氨苠青霉素作为阳 性对照;

接种物制备：大肠杆菌及金黄色葡萄球菌均取单菌 落接种于 $20 \mathrm{~mL}$ LB 培养基中，培养 $18 \sim 24 \mathrm{~h}$, 调配成 0.5 麦氏比浊标准的菌悬液.

结果判断：以在小孔内完全抑制细菌生长的最低药 物浓度为 MIC. 当阳性对照孔(即不含抗生素)内细菌明 显生长试验才有意义.

\section{References}

[1] Rahman, M. M.; Gray, A. I.; Khondkar, P.; Sarker, S. D. Pharm Biol. 2008, 46, 356.

[2] Nakahara, K.; Roy, M. K.; Ono, H.; Maeda, I.; Ohnishi-Kameyama, M.; Yoshida, M.; Trakoontivakorn, G. J. Agric. Food Chem. 2003 $51,6456$.

[3] Sohn, H. Y.; Son, K. H.; Kwon, C. S.; Kwon, G. S.; Kang, S. S. Phytomedicine 2004, 11, 666.

[4] Hirpara, K. V.; Aggarwal, A. J.; Mukherjee, N.; Joshi; Burman, A. C. Curr. Med. Chem. 2009, 9, 138 .

[5] Yoon, H.; Kim, T. W.; Shin, S. Y.; Park, M. J.; Yong, Y.; Kim, D. W.; Islam, T.; Lee, Y. H.; Jung, K. Y.; Lim, Y. Biol. Med. Chem. Lett. 2013, 23, 232

[6] Cao, W. G.; Liu, Z. Q.; Shao, Y.; Tao, Y. Z. Acta Bot. Boreal.-Occident. Sin. 2003, 23, 2241 (in Chinese). (曹纬国，刘志勤，邵云，陶燕铎，黄酮类化合物药理作用的研 究进展, 2003, 23, 2241.)

[7] Moriyasu, M.; Ichimaru, M.; Nishiyama, Y.; Kato, A.; Mathenge, S. G; Juma, F. D.; Nganga, J. N. J. Nat. Prod. 1998, 61, 185.

[8] Yenesew, A.; Midiwo, J. O.; Miessner, M.; Heydenreich, M.; Peter, M. G. Phytochemistry 1998, 48, 1439.

[9] Na, M.; Jang, J.; Njamen, D.; Tanyi Mbafor, J.; Tanee Fomum, Z.; Kim, B. Y.; Oh, W. K.; Ahn, J. S. J. Nat. Prod. 2006, 69, 1572.

[10] Promsattha, R.; Tempesta, M. S.; Fomum, Z. T.; Mbafor, J. T. J. 


\section{Nat. Prod. 1988, 51, 611.}

[11] Zuo, W. B.; Yang, J. H.; Li, H. J.; Guo, D. D.; Luo, J. S.; Huang, W. Q. Chin. J. Org. Chem. 2012, 32, 2276 (in Chinese).

(左武标, 杨金会, 李红俊, 郭冬冬, 落俊山, 黄文倩, 有机化学, 2012, 32, 2276.)

[12] Yang, J. H.; Li, H. J.; Zhang, Y. H.; Jiang, S. Z.; Li, Y. F.; Xue, P.; Ma, Y. L.; Liu, W. Y. Chin. J. Org. Chem. 2011, 31, 1230 (in Chinese).
(杨金会, 李红俊, 张玉恒, 江世智, 李云峰, 薛屏, 马玉龙, 刘 万毅, 有机化学, 2011, 31, 1230.)

[13] Yang, J. H.; Luo, J. S.; Guo, D. D.; Huang, W. Q. Chin. J. Org. Chem. 2012, 32, 1749 (in Chinese).

(杨金会, 落俊山, 郭冬冬, 黄文倩, 有机化学, 2012, 32, 1749.)

[14] Zhang, Y. H.; Yang, J. H.; Li, H. J.; Jiang, S. Z.; Li, Y. F.; Liu, W. Y. Chin. J. Chem. 2011, 29, 521.

(Li, L.; Lu, Z.) 